\title{
Educación de los cuerpos: crítica de la reproducción social y de las potencialidades de su transformación en el marco de la Educación Física*
}

\author{
Education of the bodies: a critique of social reproduction and of potential of its transfor- \\ mation in Physical Education
}
Educação dos corpos: crítica da reprodução social e das potencialidades de sua transformação no marco da Educação Física

\begin{abstract}
Emiliano Matías Gambarotta, ${ }^{a}$ Eduardo Lautaro Galak ${ }^{b}$

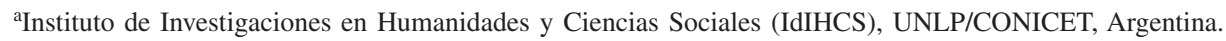
Grupo de Estudios en Educación Corporal (GEEC, UNLP). TelF.: 54221 5063950. Correo electrónico: emilianogambarotta@yahoo.com.ar

${ }^{\text {b} I n s t i t u t o ~ d e ~ I n v e s t i g a c i o n e s ~ e n ~ H u m a n i d a d e s ~ y ~ C i e n c i a s ~ S o c i a l e s ~(I d I H C S), ~ U N L P / C O N I C E T, ~ A r g e n t i n a . ~}$ Grupo de Estudios en Educación Corporal (GEEC, UNLP) y del Grupo de Estudios sobre Sociología de las Emociones y los Cuerpos (IIGG-UBA). Telf.: 54221 4274341. Correo electrónico: eduardogalak@gmail
\end{abstract}

\begin{abstract}
Este escrito da cuenta de los mecanismos claves en la reproducción del orden social establecido, particularizando la mirada en aquellos que se ponen en acto en la Educación Física, cuestión que también entraña reflexionar sobre la educación en general y sobre la sociedad moderna. En este marco, nos enfocaremos en el caso argentino, interrogándonos por -lo que con Bourdieu llamamos- el entrelazamiento de nomos y doxa que allí se establece, como por la dominación simbólica que se produce. Todo lo cual nos llevará a analizar los modos en que se concibe esta disciplina y el espacio que ocupa en la currícula como una instancia de control de aquello que podría "descontrolar" la educación intelectual; sin embargo, veremos también como esto conduce a un des-control de dicho control. Es dando cuenta de estos mecanismos, practicando su crítica, que podemos aspirar a transformarlos, para así buscar maneras de transformar las relaciones sociales y desandar los caminos de su reproducción.
\end{abstract}

RESUMEN

Palabras clave: reproducción social, educación física, Argentina, dominación simbólica, educación corporal.

\begin{abstract}
This paper aims to analyze key mechanisms of social order reproduction, specifically in Physical Education; an issue that also involves thinking about education in general and on modern society. In this sense, we focus on the Argentinian case, questioning what Bourdieu called the intertwining of nomos and doxa, and the symbolic domination that it produces. This allows us to analyze the ways in which this discipline is conceived and the space it occupies in the curriculum as an instance of control of what could "de-control" the intellectual education; nevertheless, it also permits to see how this produces a "de-control" of that same control. It is by thinking these mechanisms and practicing its criticism, that we can conceive the ways to transform them, and the ways to retrace the paths of its reproduction.
\end{abstract}

Key words: social reproduction, physical education, Argentina, symbolic domination, corporal education.

* Este artículo fue solicitado por la Revista Estudios Pedagógicos en diciembre del 2011 en el contexto del proyecto de Investigación FONDECYT (Fondo de Investigación Científica y Tecnológica) No 11110016 , titulado "Educación Física y su función de transformación de las desigualdades sociales: profesorado del área y documentación ministerial". El artículo fue aceptado en junio de 2012. El mismo ha sido posible gracias al financiamiento recibido del Consejo Nacional de Investigaciones Científicas y Técnicas (CONICET) de la República Argentina, del cual ambos autores son becarios. 


\section{RESUMO}

Analisam-se os principais mecanismos de reprodução da ordem social estabelecida, particularmente aqueles praticados em Educação Física, questão que também precisa ser analisada na educação e na sociedade, de forma geral. Neste sentido, é enfocado o caso da Argentina e que, conforme Bourdieu, denominamos de entrelaçamento de nomos e doxa que ali se estabelece dada a dominação simbólica que se produz. Tudo isto leva à análise das maneiras como esta disciplina é concebida e o espaço por ela ocupado no currículo como meio de controle do que poderia "descontrolar" a educação intelectual, mas que conduz a um descontrole de tal controle. Compreendendo tais mecanismos, praticando sua crítica, pode-se aspirar à transformação de eles para, assim, encontrar formas de transformar as relações sociais e desfazer os caminhos da sua reprodução.

Palavras chave: reprodução social, educação física, Argentina, dominação simbólica, educação corporal.

\section{INTRODUCCIÓN}

En este trabajo nos interrogaremos sobre el modo en que la Educación Física puede constituir un momento de la reproducción del orden social establecido. Más específicamente, nos preguntaremos acerca de si en la Educación Física pueden hallarse elementos tendientes a la (re)producción de las relaciones de dominación imperantes y, de ser así, cómo es que tales elementos se ponen en juego. Aclaremos inmediatamente que si bien nuestras indagaciones se centrarán en la Educación Física y, dentro de ella, en la Educación Física argentina, consideramos lo planteado para ésta como una vía por la cual reflexionar sobre la educación en general; en definitiva, procederemos mediante sinécdoque.

Ahora bien, los interrogantes antes mentados no pueden aprehenderse más que percibiendo la práctica de la Educación Física sobre el telón de fondo del entramado relacional en su conjunto y, en última instancia, sobre una tematización de cómo la sociedad se da un orden a sí misma. De allí que dediquemos la primera sección a establecer dicho telón de fondo, para lo cual nos apoyaremos en la concepción elaborada por Pierre Bourdieu, pues su perspectiva permite indagar densamente los sentidos que los agentes ponen en juego en sus prácticas, sin por ello descuidar no sólo sus consecuencias objetivas, sino también la interrogación reflexiva acerca del modo en que las relaciones sociales establecidas impactan en las categorías a través de las cuales se producen tales sentidos. Cuestión en la cual puede notarse el particular entrelazamiento entre una dimensión cognitiva y una atinente a lo político que subyace al planteamiento bourdieuano.

Sin embargo, cabe destacar que no concebimos este retomar la concepción bourdieuana como una suerte de marco teórico que meramente vamos a "aplicar" en nuestro trabajo, antes bien, es sólo preguntándonos primero por cómo se (re)produce el ordenamiento social que podemos interrogarnos por los posibles mecanismos que la Educación Física entraña y hacen de ella un momento de tal reproducción. A la vez, esto constituye una instancia clave de todo intento de "hacer saltar por los aires" tales mecanismos, ya que la captación de los mismos puede fungir como un paso (no necesario, mas no por ello desdeñable) de la práctica orientada a su transformación. Con lo cual nuevamente nos hallamos ante el entrelazamiento de lo cognitivo y lo político que recorrerá el conjunto de este escrito, pues el modus operandi que aquí ponemos en juego -a partir de una particular apropiación del pensamiento de Bourdieu- tiene en ese entrelazamiento una de las instancias centrales a través de las cuales se interrogan los procesos socio-históricos y culturales. Pero, también por su centralidad para pensar el rol de la educación (física) en la reproducción del ordenamiento (político) de lo social, por lo que la dimensión atinente a lo político de la práctica cognitiva, que en última instancia también este escrito 
contiene, no es una mera declaración de "buenas intenciones", sino una parte integrante del modo en que se configura y se aborda la problemática a ser indagada.

Cabe destacar también que la pregunta por el "cómo" de estos mecanismos de (re) producción que aquí nos hacemos (tanto en lo referente a la Educación Física como al ordenamiento de lo social en su conjunto) apunta a dar cuenta de los modos en que ellos tienen lugar, de la constelación de factores que los configuran, poniendo en juego un pensamiento relacional que busca sortear las trampas de todo substancialismo. Es por ello que nuestra perspectiva hace foco en el modo de (re)producción del ordenamiento social, dentro del cual aquí nos interesará específicamente el modo de (re)producción de los cuerpos, en tanto instancia clave de aquél. Desde este punto de vista, construiremos nuestro argumento en torno a la Educación Física argentina, interrogándonos acerca de la educación en general, acerca de los elementos que en ella contribuyen a la reproducción de la sociedad moderna y sus relaciones de dominación pero también del potencial de la educación para transformar aquello que reproduce.

\section{DOMINACIÓN SIMBÓLICA Y DOBLE NATURALIZACIÓN DE LO SOCIAL}

La clave del pensamiento bourdieuano sobre la reproducción del ordenamiento social está dada por su particular concepción del plano simbólico; es en ese terreno que se enfoca nuestro análisis. Tal plano conforma un "tercer orden", como diría MerleauPonty (1957), en tanto no es ni puramente objetivo ni puramente subjetivo, sino que se encuentra en la ambigüedad entre ambos, en la relación entre los habitus de los agentes y el campo en cuestión. Es decir, entre las disposiciones de percepción, apreciación y acción incorporadas por tales agentes, como producto de sus posiciones objetivas en ese campo (definida por el volumen y composición de capitales que hayan acumulado), pero que también es producto de la propia historia de esas posiciones, esto es, de sus trayectorias en el interior de un campo. De esta manera, la noción de habitus nos remite al entramado relacional que tales posiciones configuran, pues es en el vínculo entre todas ellas que el campo adquiere su particular estructura.

Entonces, en esta relación entre habitus y campo hunde sus raíces la captación del plano simbólico planteada por Bourdieu o, más específicamente, dicha captación se enraíza en el entrelazamiento de nomos y doxa. La primera de ellas refiere al punto de vista constitutivo de un campo, a su ley fundamental que es también su principio de visión y de división; ${ }^{1}$ a partir del cual se establece la taxonomía social que divide en grupos y que contribuye a que se los conozca y reconozca como tales. Nomos está en permanente relación con aquello que Bourdieu denomina la doxa, el conjunto de presupuestos cognitivos y evaluativos que un agente posee y acepta implícitamente por el hecho de pertenecer a un determinado campo, presupuestos que tienden a producir una adhesión inmediata al nomos de ese campo (el cual, a su vez, al ser el punto de vista constitutivo

$1 \quad$ Bourdieu sostiene que "nomos viene del verbo nemo, que quiere decir operar una división, una partición; comúnmente es traducido por ley, pero también es, más precisamente, lo que llamo el principio de visión y de división fundamental característico de cada campo" (2001: 18). En este sentido, Bourdieu (1999: 129) aclara que "constitución" es más exacto que "ley" para definir nomos; sin embargo, se deja esta acepción para dar cuenta de su sentido lato. Cf. Bourdieu, 2001: 37-38; Galak, 2010: 120. 
del campo es un factor fundamental en la estructuración de tales presupuestos cognitivos y evaluativos). Vemos cómo estas categorías no pueden ser percibidas más que en su entrelazamiento, en su constante movimiento que lleva a que una de ellas nos remita a la otra y viceversa. Pues si bien puede considerarse que el nomos remite primordialmente al campo y, con éste, a lo objetivo, su fuerza reside en ser una instancia central de la configuración de los presupuestos cognitivos que los agentes ponen en juego en sus prácticas. Y si en este sentido la doxa remite a lo subjetivo, lo relevante de esta noción es que nos permite aprehender también las consecuencias objetivas que tales presupuestos generan, cómo esto impacta en la reproducción del nomos establecido y, con éste, del actual estado del entramado relacional en su conjunto. ${ }^{2}$ Es por esto último que si bien estas categorías son relativas a una determinado contexto socio-histórico y cultural -a lo cual se agrega su estar vinculadas a una determinada posición social-, no han de verse sus consecuencias como tan fácilmente relativizables; en efecto, el mundo social "con sus jerarquías que se resisten a dejarse relativizar tan fácilmente, no es relativista" (Bourdieu, 1999: 103). Pues aun cuando los modos de dotación de sentido que subyacen a una relación de dominación sean relativos, no por ello lo es la violencia que se ejerce sobre los dominados. ${ }^{3}$

A partir de esto podemos plantear que la reproducción del ordenamiento social y, con él, de las relaciones de dominación (simbólicas) que allí tienen lugar, se asienta principalmente en el proceso (socio-histórico) por el cual se transmuta "la arbitrariedad del nomos social en necesidad de la naturaleza" (Bourdieu, 2007: 26). Es decir, a través de una "naturalización" de lo socio-histórico que, como tal, diluye su carácter de, justamente, socio-histórico; "trabajo histórico de deshistorización" (Bourdieu, 2007: 104-105) por el cual se percibe como eterno lo que es un producto de prácticas históricas, como dotado de una substancia en sí lo que surge de relaciones sociales. Esto es, en una reactualización del precepto marxista ${ }^{4}$ según el cual la naturalización entraña el olvido

2 Una tercera noción que, en el pensamiento bourdieuano, completa este vínculo surge de la noción de illusio; ya que es el entrelazamiento entre nomos y doxa el que dota de sentido al juego, pero también lo dota de su valor, de que valga la pena jugarlo y apostar en él. Es a esto a lo que alude la illusio, en tanto "adhesión colectiva al juego que es a la vez causa y efecto de la existencia del juego" (1995: 253). De allí que poner en cuestión esa illusio es también cuestionar la doxa y el nomos del campo, es poner "en tela de juicio no una manera de jugar el juego, sino el propio juego y la creencia que lo fundamenta, única trasgresión inexpiable" (1995: 256-257).

3 En este sentido Bourdieu señala, por ejemplo, el carácter relativo, propio de un arbitrario cultural, de la "dominación masculina" (por dar el ejemplo al que Bourdieu dedica su libro llamado, justamente, $L a$ dominación masculina) pero eso no relativiza las consecuencias que esa dominación entraña: la violencia de género y la situación de sojuzgamiento en la que muchas mujeres se encuentran.

$4 \quad$ En efecto, el uso de la noción de naturalización por parte de Bourdieu tiene una de sus fuentes principales en la tradición marxista, especialmente en la vertiente crítico-dialéctica de raíz alemana y en la noción de "segunda naturaleza" que allí se pone en juego como parte (central) de su crítica a las sociedades modernas. Ya que, a través de ella, se critica la petrificación del movimiento socio-histórico, a la vez que se señala cómo el producto de las prácticas de los agentes sociales, la sociedad, es percibida como dotada de una lógica propia, extraña a dichos agentes que, por tanto, no pueden modificarla con su actuar. En definitiva, la noción de "segunda naturaleza" no sólo alude al proceso histórico de eternización, sino también a cómo lo social se presenta como regido por una necesidad inmodificable ante la cual no queda más que una resignación en la práctica. Tal es, por ejemplo, el modo en que Lukács la define -en una de las primeras utilizaciones de esta noción- al sostener que, en la actualidad, el mundo social "es una segunda naturaleza; como la primera, no puede ser definida sino como un sistema de necesidades conocidas, pero cuyo sentido permanece extraño" (Lukács, 1971: 66). 
de la historia, Bourdieu sostiene que, para escaparle a "la" historia, paradójicamente no hay otra manera que practicando un proceso de historización (Bourdieu, 1992: 38) En definitiva, la percepción como "natural" de los procesos socio-históricos los sitúa más allá del alcance de los agentes y sus prácticas, otorgándoles un carácter fatal a las relaciones establecidas, a los modos de dominación allí imperantes.

De esta manera, el entrelazamiento entre nomos y doxa lleva a una naturalización tanto de las categorías con las que los agentes perciben al entramado relacional, como de este mismo entramado relacional, en el cual los agentes realizan sus prácticas. Por lo que estamos ante un proceso que no tiene lugar sólo en el ámbito objetivo, o sólo en lo subjetivo, sino en ambos a la vez, y en esto reside, entonces, "uno de los mecanismos más poderosos del mantenimiento del orden simbólico, a saber, [en] la doble naturalización que resulta de la inscripción de lo social en las cosas y los cuerpos" (Bourdieu, 1999: 238). Este mecanismo, cuya fuerza tiene una de sus manifestaciones más claras en lo que Bourdieu denomina el "sentido de los límites" (Bourdieu, 1999), es el medio por el cual se percibe y aprecia una determinada toma de posición como por fuera de las posibilidades prácticas de los agentes, como imposible de ser realizada por ellos. Es esto lo que subyace -en última instancia- a esa frase que constituye una suerte de leitmotiv de su obra: "esto no es para nosotros". Resignación práctica y de la práctica, ya que, por un lado, acontece no como toma de decisión consciente (al estilo de los planteos de la "Teoría de la Elección Racional") sino como producto de un sentido práctico incorporado por los agentes sociales. Esto, a la vez, entraña una clausura de aquellas prácticas que, al atentar contra lo que esta doble naturalización establece, son vivenciadas como "anti-naturales", por lo que ni siquiera vale la pena intentar concretar semejantes tomas de posición. Todo lo cual refuerza el carácter fatal con que es percibido el orden simbólico establecido.

Más aún, la doble naturalización de lo socio-histórico no sólo obtura la emergencia de prácticas tendientes a transformar esa lógica relacional, sino que también obstaculiza la aprehensión de la posibilidad misma de tal transformación. En efecto, ni siquiera puede aspirarse a modificar aquello que no es percibido o problematizado como modificable; de allí la centralidad, para la transformación práctica de las relaciones de dominación imperantes, de transformar no sólo las "cosas", la estructura de posiciones sociales, sino también los "cuerpos", las estrategias de dotación de sentido incorporados por los agentes. Esto último nos sitúa, una vez más, ante el entrelazamiento de lo cognitivo y lo político, al plantearse las consecuencias en lo político de las categorías a través de las cuales los agentes dotan de sentido al mundo social y viceversa, las consecuencias cognitivas de la forma en que la sociedad se da un orden a sí misma.

Es en este plano, entonces, donde se enraíza nuestra preocupación por los principios de visión y división puestos en juegos en la educación y, más específicamente, en la Educación Física argentina. En tanto los procesos educativos constituyen una de las instancias claves de esta reproducción de un orden simbólico -que tiene entre sus pilares fundamentales la adherencia entre lo cognitivo y lo político-, dada la centralidad de la institución escolar en las sociedades modernas. El modo bourdieuano de interrogar estas problemáticas que aquí ponemos en juego, nos permite aprehender esto no como respondiendo a prácticas conscientes de agentes de peso en el diseño de los currículos, ${ }^{5}$ o bien

Aclaremos que esto tampoco implica negarle todo lugar a la acción consciente de tales agentes, sino indagar a éstas como parte del conjunto de acciones posibles, mas no las únicas. 
como un objetivo más o menos explícito en los programas de las distintas asignaturas, sino como una lógica subyacente a ello, como en definitiva producto del entrelazamiento de nomos y doxa que allí tiene lugar. El cual también naturaliza para los agentes productores de esos currículos, o de esos programas, los principios de visión y de división allí puestos en juego, contribuyendo así a la reproducción práctica de tales principios a través de uno de los mecanismos más eficaces posibles: aquél que ni siquiera es percibido como tal, pues no podemos pensarlo más que mediando las mismas categorías que ese mecanismo pone en movimiento.

Llegamos así a interrogarnos por la "violencia simbólica" que aquí se ejerce y por la dominación a que ella da lugar. Ambas son producto del desconocimiento práctico generado por la implicación en el mundo social de los agentes sociales, que lleva a aceptar como evidentes y "naturales" no sólo el modo en que se juega un determinado juego social sino al juego mismo. Sin embargo, la dominación simbólica no es un mero desconocimiento, también entraña el reconocimiento práctico que produce una manera de dotar de sentido al mundo, asentada en el desconocimiento del carácter relativo y arbitrario del orden social. Así,

la violencia simbólica es esa coerción que se instituye por mediación de una adhesión que el dominado no puede evitar otorgar al dominante (y, por tanto, a la dominación) cuando sólo dispone, para pensarlo y pensarse o, mejor aún, para pensar su relación con él, de instrumentos de conocimiento que comparte con él y que, al no ser más que la forma incorporada de la estructura de la relación de dominación, hacen que ésta se presente como natural (Bourdieu, 1999: 224-225).

Sin embargo, si bien la dominación simbólica implica esa particular adhesión del dominado, lo que ella entraña "no es sumisión pasiva a una norma externa ni adhesión libre a valores" (Bourdieu, 2008: 29), antes bien "se inscribe en la práctica, en las disposiciones que se inculcan poco a poco, a través de un proceso de adquisición largo y lento" (Bourdieu, 2008: 30). Se enraíza, en definitiva, en una dimensión práctica, que tiene uno de sus pilares fundamentales en principios de visión y división incorporados por los agentes sociales, vueltos cuerpo. Es en este marco que cobra toda su centralidad la interrogación que aquí nos hacemos acerca del modo de (re)producción de los cuerpos que la Educación Física argentina pone en acto, de cómo en ella se configura una constelación de elementos que instauran una particular modalidad de corporalidad. En este punto se enfoca nuestro interés, pues es dando cuenta de estos mecanismos de reproducción que podemos realizar una contribución (no imprescindible, pero no por ello desdeñable) a la disrupción de tales mecanismos. En definitiva, es movilizando socio-históricamente lo que la naturalización petrifica y eterniza que podemos aspirar a transformarlo.

\section{DE LA educación física A LA Educación Física}

Aquella constelación de elementos que conforma modos particulares de la Educación Física, a la que hacíamos referencia líneas atrás, se pone de singular manera en evidencia en los procesos constitutivos que dieron origen a la misma. Esto es, en los sucesos que provocaron como consecuencia las primeras configuraciones de qué se produjo bajo el nombre de la disciplina pueden encontrarse los argumentos que explican cómo ella (re) produce particulares modos del cuerpo. 
Sin embargo, antes de continuar con este desarrollo, conviene partir de dos aclaraciones preliminares que reflejan, además, puntos de partida epistémicos: un primer elemento que nos permite observar la emergencia de un nomos puede ser encontrado en la distinción entre educación física con minúsculas y Educación Física con mayúsculas, entendiendo que la primera implica una manera de denominar actos pedagógicos que comprometen la utilización manual o corporal; mientras que la segunda expresión apunta a nombrar aquellas prácticas que homogénea, regular y sistemáticamente hayan sido institucionalizadas como "Educación Física". Dicho de otro modo, mientras que la primera refiere a las instrucciones que aluden y utilizan al cuerpo, la segunda remite sólo a las prácticas de la pedagogía institucional del cuerpo por excelencia (Rodríguez Giménez, 2010). Es decir que si bien la disciplina Educación Física encierra ambas prácticas, es la segunda la que entraña el reconocimiento de ciertos saberes (y no otros) como válidos, legitimándolos como materia dentro de los emergentes Sistemas Educativos argentinos. Es en el pasaje de una instancia a la otra donde pondremos el foco, no sólo porque allí pueden observarse las disputas en pos del dominio simbólico sobre la disciplina, sino sobre todo porque esto nos permitirá aprehender los rasgos predominantes que la Educación Física argentina adquiere en su génesis, el modo de producción de sentido que se instaura, pero también el que (re)produce con su práctica. Veremos, así, los primeros hilos, claves en tanto que genéticos, de la trama de sentido que allí se teje, de los principios de (di)visión que resultaron triunfantes.

Los procesos cuyo desenlace producen strictu sensu el nacimiento de la disciplina pueden datarse con precisión en la década de 1880, punto neurálgico por la sanción de la Ley de Educación Común n 1.420 que promulga las bases para la creación de los Sistemas Educativos Nacionales en Argentina, desarrollando así un Estado-Docente (Narodowski \& Manolakis, 2001: 29) e instaurando como pensamiento político-pedagógico, en el mismo proceso y procedimiento, la promoción de una educación integral: intelectual, moral y física. Precisamente en este punto encontramos el segundo elemento que nos permite observar la emergencia del nomos constitutivo de la disciplina, y que es a la vez el segundo posicionamiento epistémico que explicitamos: partimos de comprender que la educación tal como la concebimos hoy en día es un producto eminentemente moderno y que, como tal, pone en juego, sino todos, algunos de los más importantes principios de (di)visión que caracterizan al pensamiento moderno. En ese contexto, a raíz del ideario pedagógico de la época que entendía que la educación debía ser intelectual, moral y física, surge la Educación Física, disciplina hija de los Sistemas Educativos nacionales, "nacida de la Europa de fines de siglo XIX del vientre de la ciencia y de la mano del Estado" (Crisorio, 2009: 48). Esto es, como parte del juego, la educación física jugó el juego que le correspondía según el ideario de que debía formar parte de la educación integral del hombre, tal cual quedaría plasmado con la Ley de Educación Común 1.420. Con esto nos distanciamos de aquellos investigadores que encuentran la génesis de esta disciplina en las formas originarias de movimientos, con ciertos rasgos sistemáticos. Es decir, de quienes la sitúan en la existencia, por caso, de una continuidad y contigüidad lógica con el movimiento olímpico de la Grecia clásica o con los juegos tradicionales aborígenes. Antes bien, entendemos que para el caso argentino la "Ley 1.420" pone en cuestión, en primera instancia, la educación física y, como consecuencia, sirve de base para la construcción de la Educación Física propiamente dicha. 


\subsection{PROCESOS DISRUPTIVOS Y REPRODUCCIONES: LEGISLACIÓN Y EDUCACIÓN FÍSICA}

Veamos, pues, la legislación, y en detalle sus artículos referidos a la educación de los cuerpos. La "Ley de Educación Común" estableció una serie de lineamientos generales que dieron origen y enmarcaron los Sistemas Educativos en Argentina. Firmada el 8 de Julio de 1884 por el entonces Ministro de "Justicia, Culto e Instrucción”, Eduardo Wilde, y por el Presidente Julio Argentino Roca, esta normativa expresa la convergencia de tres procesos contemporáneos: por un lado, los principales postulados que sostenía el proyecto político de la llamada "Generación del 80", por ese entonces gobierno de las ideas y del país; por otro, el desarrollo y articulación con su impronta moderna de instituciones estatales encargadas de la educación común de sus ciudadanos, proceso que paralelamente se desarrollaba, con sus matices, en todos los Estados-Nacionales emergentes; y, por último, la puesta en práctica de las conclusiones obtenidas como resultado de los debates en el "Congreso Pedagógico" que tuvo lugar en 1882, aunque no sin disputas. Respecto a esta última, existe una continuidad ideológica y política que atravesó tanto el Congreso cuanto la Ley: hacemos referencia a que en ambas instancias primó una razón del hombre cabal cuya educación integralista debía contemplar lo intelectual, lo moral y lo físico; sumado a una justificación pedagógica basada en criterios científico-positivistas laicos en reemplazo de los juicios de verdad eclesiásticos. Sin embargo, como decíamos, estas ideas no estuvieron exentas de disputas: la confusión entre intereses de la elite e intereses del país que postulaba el ideario ochentista se pone de manifiesto en la pretensión de perdurar a través de la educación ciertos saberes (y no otros). Tal cuestión se reproducirá tanto en la organización de los Sistemas Educativos cuanto para la estructuración de, primero, la educación física, repercutiendo, luego, en la Educación Física. En efecto, antes de 1884 (aunque en ciertas formas también después) la educación física en las escuelas era un conglomerado de ejercicios gimnásticos, ejercitaciones militares y actividades físicas y recreativas que alternaban asistemáticamente entre lo obligatorio y lo opcional, entre las materias con horarios fijos y los recreos. Por ello, no resulta extraña la proposición en el Congreso Pedagógico del Dr. Honorio Leguizamón, médico y rector del Colegio Nacional de Concepción del Uruguay, en cuyo discurso convergen los sentidos que justificaban la educación física como parte de la escuela:

no puedo asentir como higienista, a que la enseñanza física sea obligatoria solamente para las escuelas comunes. Es una necesidad social propender al desarrollo físico de las razas; y por consiguiente, tanto en las escuelas comunes como en las particulares, es precisamente donde el Gobierno, el Estado, la sociedad, deben hacer sentir sus esfuerzos y su influencia para la realización de ese propósito. Creo pues, que la enseñanza de las evoluciones y ejercicios militares -indispensables para niños que mañana van a ser miembros de una sociedad que se halla en el caso de concurrir con todos sus medios al desarrollo físico y vigor de nuestra raza-está perfectamente indicada para ser obligatoria en todas las escuelas (cit. en Saraví Riviere, 1985: 75).

En todo esto podemos encontrar una convergencia de intereses entre política y pedagogía que se evidencia en las conclusiones del Congreso Pedagógico sobre educación física escolar y que fueron refrendadas en la Ley 1.420. Respecto al primero, Saraví Riviere (1985: 76-77) enumera como principales resultados sobre la asignatura los siguientes puntos: los congresales a) establecieron de común acuerdo que la educación 
perseguida debía ser completa, física, intelectual y moral; b) incluyeron a la educación física como materia indispensable de la escuela primaria, y, en consecuencia, obligatoria; y c) la limitaron al sólo comprenderla como gimnástica. ${ }^{6}$ Ahora bien, conviene realizar al respecto tres salvedades: en principio, la referencia es exclusivamente a la escuela primaria (el análisis de la educación media, industrial y profesional sería tema de discusión del frustrado Segundo Congreso Pedagógico a desarrollarse en 1885); como segunda cuestión cabe remarcar que la sinonimia en la interpretación de la materia como gimnástica se hallaba oculto el temor de confundir -fundamentalmente para el caso de los varones- actividades físicas con ejercicios militares $;^{7} \mathrm{y}$, por último, que el postulado de los congresistas que pretendían hacer de la educación física una materia obligatoria quedó completamente solapado en la Ley de 1884, al punto que, como tal, no figura. Sin embargo, en los artículos $1^{\circ}$ y $14^{\circ}$ de la Ley podemos encontrar una doble dimensión que signa el accionar de la asignatura desde sus orígenes y que puede vérsela, con distintas intensidades y frecuencias, hasta la actualidad: por un lado, el lugar secundario otorgado, producto de ser concebida como complemento de la educación integral y a raíz de la distinción entre un tronco básico escolar (dictado clases diarias áulicas) de otro cúmulo de asignaturas accesorias, y, por el otro, la adherencia a los nuevos Sistemas Educativos de discursos que apunten a educar al cuerpo, más no sea como recurso para conseguir utilidad intelectual.

El artículo $1^{\circ}$ de la Ley de 1884 plantea que "la escuela primaria tiene por único objeto favorecer y dirigir simultáneamente el desarrollo moral, intelectual y físico de todo niño de seis a catorce años de edad". Esta búsqueda por una educación integral, presente en la mayoría de los textos pedagógicos argentinos de la época, encuentra eco en la doctrina positivista educativa justificada en el libro Educación intelectual, moral y física de Herbert Spencer; y pone de manifiesto la marcada confluencia entre los estudios de la biología, la psicología y la pedagogía, en el contexto del modo de dotación de sentido predominante que signaba a la época. De esta manera, el niño debía desarrollar en su escolarización la tríada educativa en completa armonía con la evolución -argumentada en

6 Resulta llamativo que los juegos no se mencionan ni una sola vez, aunque estaba presente en el ideario de la época (Pourteau, 1897). Distinto es el caso de los deportes que, como es sabido, comenzó a ser discutido como contenido escolar recién entrado el primer cuarto de siglo XX.

7 Temor en absoluto infundado: el primer centro de formación de profesionales en el área fue la Escuela de Gimnasia y Esgrima del Ejército argentino, fundada en 1897 (Cristiani, 1967), reflejando así tanto la confusión entre educación física y gimnasia cuanto la de actividades físicas con ejercicios militares. De hecho, el propio Domingo Faustino Sarmiento llamó a la “gimnasia militar" el “germen de la institución del porvenir" (Sarmiento, 1900). Recién en 1901 comenzaría a verse plasmado un movimiento político-pedagógico para establecer una profesionalización no-militar con la instauración de los "Cursos de ejercicios físicos", que, de la mano del Dr. Enrique Romero Brest, considerado el "padre de la Educación Física argentina” (Dallo, 2007; Aisenstein y Scharagrodsky, 2006, Scharagrodsky, 2004), "el Sarmiento de la educación física” (Scharagrodsky, 2010), funcionaron en cinco oportunidades de manera temporaria hasta que en 1906 se establecieran permanentemente como "Escuela Normal de Educación Física", pasando a llamarse, en 1912, como "Instituto Nacional", denominación que sobrevive hasta la actualidad (Romero Brest, 1903). La biografía del Dr. Enrique José Romero Brest es particularmente importante para la historia de la disciplina: diseñó el "Sistema Argentino de Educación Física", método oficial de enseñanza de la disciplina durante las primeras tres décadas del siglo XX, se doctoró en medicina en la Universidad de Buenos Aires, fue autor de una extensa bibliografía y director desde su creación hasta la década de 1930 del "Instituto Nacional Superior de Educación Física", que actualmente lleva su nombre a modo de homenaje. 
criterios científicos- ${ }^{8}$ psico-motriz correspondiente. En síntesis, la propuesta pedagógica integralista spenciariana apuntaba a una educación que emancipe, que puede reproducirse de generación en generación y que sea justificada por conocimientos científicos. En este sentido, Spencer pone de manifiesto una visión organicista (compatible y complementaria del evolucionismo y del "Darwinismo Social") que refuerza el modelo dualista del ser humano que asemeja al cuerpo físico y sus funciones con las de los animales, aunque sin reducir su análisis a "puro biologicismo". Es decir, al incluir, por ejemplo, la vestimenta o la distinción de educación para niños de para niñas, Spencer demuestra que no debe limitarse la enseñanza de lo físico a una supuesta reproducción de la naturaleza, sino que, aún cuando reduce el cuerpo a lo biológico, entiende que existe una potencia social de la educación que permite interpelar, instruir, modificar. Más aún, comprendía que la principal función de la misma es "formar un ser apto para gobernarse a sí mismo, no un ser apto para ser gobernado por los demás" (Spencer, 1946: 206); lo cual nos permite desarrollar uno de los principales sentidos que promueve el entrelazamiento de nomos y doxa que tiene lugar en la Educación Física y que ponemos en juego como uno de los ejes centrales de discusión: esta noción de "gobierno de sí mismo" está particularmente patente en las prácticas de la disciplina, se halla en la matriz de sus justificaciones y razones para su inclusión en los Sistemas Educativos, y ello porque esta asignatura apunta a controlar aquello que se descontrola. Esta cuestión encuentra razón en la relación entre el trabajo mental y el manual que la educación integralista puso de manifiesto en los Sistemas Educativos argentinos: la educación física, como complemento de la intelectual y moral, resulta ser una descarga de las tareas áulicas, y por ello se hace análoga con los recreos. Es decir, el argumento que permite el ingreso de la educación física a los Sistemas Educativos halla su justificación en que, mediante el gobierno del cuerpo, permitía la compensación del gasto energético necesario para la instrucción intelectual y favorecía la formación del carácter que la educación moral precisa.

Esta cuestión puede verse a las claras en la consigna de Spencer (1946: 272-273), según la cual la educación física debía subsanar el "exceso de aplicación mental"; se actualiza en el debate, entonces, en la Educación Física, acerca de lo que Romero Brest llamó el "proletariado intelectual". En ese marco, planteó los inconvenientes que provocaba el desarrollo de una educación "puramente" intelectual, tal como "es el concepto práctico dominante de muchos educadores" (INSEF, 1923: 10), renovando así la crítica a la escolarización por el interés unilateral hacia lo intelectual, "descuidando casi de una manera total, toda otra forma de disciplina que no tenga relación directa con el desarrollo de las aptitudes psíquicas" (Romero Brest, 1913: 9). ${ }^{9}$ Sin embargo, Romero Brest alertaba sobre la posibilidad de que sin una "integración utilitaria" podría crearse un "proletariado

\footnotetext{
En efecto, ante la pregunta de cuál es el saber más útil, el intelectual, el moral o el físico, Spencer (1946: 82) argumentaba que es "la ciencia".

9 Si bien por ese entonces la palabra de Romero Brest era sin lugar a dudas una de las más autorizadas en el ámbito de la Educación Física, cierto es que en aquella época en la que se denunciaba el exceso de intelectualismo la disciplina se encontraba en pleno debate entre posturas más ligadas a la pedagogía civil y otras emparentadas con la corporación militarista. Sin embargo, a pesar de sus múltiples diferencias respecto a la disciplina, coincidían en declamar el utilitarismo que se le otorgaba a la educación del cuerpo. Claro está, la "tradición romerista" lo denunciaba en tanto que la "tradición militarista" pretendía profundizarla (fundamentalmente a partir de entender la educación de los niños como instrucción de "pre-conscriptos" y de pretender (re)incorporar la enseñanza del tiro en las escuelas).
} 
manual, como la escuela intelectualista creó el proletariado intelectual, por las mismas causas de desequilibrio social entre oferta y demanda" (INSEF, 1923: 10-11). Esto es, consideradas aisladamente las partes educativas que componen la educación integral, la Educación Física construyó sus discursos a partir de situarse en el legado de la educación física como producto "inferior" de la descomposición de la educación.

Todo esto nos da pie para desarrollar el segundo de los artículos de la Ley 1420, que expresa un sentido sobre la educación física: el $14^{\circ}$, el cual promulga que "las clases diarias de las escuelas públicas serán alternadas con intervalos de descanso, ejercicio físico y canto". Este refleja, como puede verse, el principio de que la educación física junto con el recreo y la educación artística, funcionan como complementos de un núcleo básico de asignaturas. Esto es, la centralidad que ocupan algunas materias en las currícula, tanto histórica como actualmente, manifiestan, a su vez, que la matriz del Sistema Educativo está en las aulas, preponderancia que refleja, evidenciando la pervivencia de ciertos dualismos, el mayor espacio otorgado a lo espiritual o a lo intelectual antes que a lo corporal. Así, la educación del cuerpo, secundaria en todos los órdenes pedagógicos, es condición primaria de todos sus sentidos (volveremos sobre esta articulación entre control y des-control que la Educación Física pone en juego más adelante). En otras palabras, este artículo de la Ley producirá un elemento que se reproducirá hasta la actualidad: la sumisión del cuerpo en dualismos, sea con la mente, el alma o el espíritu. Tal como se puede observar en el caso cartesiano cuerpo-mente o en la fórmula natural-artificial, la distinción "educación del cuerpo"-“educación del alma" nos permite reflexionar sobre lo que con Bourdieu (2007: 232-233) hemos denominado la (di)visión social del mundo -esto es, la operación de los esquemas de clasificación en forma binaria, que dan la apariencia de un fundamento objetivo a los juicios, instaurándolos como nomos, los cuales se realizan en los cuerpos, en las doxas que sostienen a tales juicios, instancias que (se) producen como resultado (de) la realidad social-, es decir la institución de modos duales antagónicos sobre la que se construye el mundo.

Volviendo al caso argentino, podemos observar en las argumentaciones de Sarmiento ${ }^{10}$ acerca de por qué debía incluirse la gimnástica en las escuelas una serie de elementos claves para el presente análisis: a) porque éstos eran un importante "factor de disciplina social, capaz de sublimar los impulsos primitivos y desarmónicos del niño y educarlo moralmente"; b) porque beneficiaría la salud del cuerpo y del alma de "todo el pueblo trabajador"; y c) porque, como hemos venido sosteniendo, complementaría la concepción integralista de la educación (Saraví Riviere, 1985: 31-42). Es decir, para Sarmiento la educación física resultaba una necesaria fuente de disciplinamiento físico, pero también moral e intelectual -de allí la sugestiva frase que rezaba que "la gimnástica civilizará á los Tobas, que no conocen disciplina sino cuando van á la guerra, á fin de robar y matar con éxito" (Sarmiento, 1900: 278)-. Tal como puede observarse, en los intersticios de la matriz de pensamiento que justificaba el ideario pedagógico de la época está presente tanto la educación integralista como modo de concebir a los sujetos y su instrucción, cuanto la utilidad de la misma para el control de los impulsos, de las pasiones, de la naturaleza, del cuerpo; cuestiones que se resumen en la constante búsqueda por producir

10 Cabe recordar que Sarmiento tuvo un papel clave para la argumentación pedagógica de los "ideales ochentistas", tanto desde su rol de "Superintendente General de Escuelas" cuanto en el de Presidente Honorario del Congreso Pedagógico de 1882. 
una identidad nacional, que es también (o sobre todo) producir un orden social y las condiciones de su reproducción.

\subsection{PROCESOS DISRUPTIVOS Y REPRODUCCIONES: CONQUISTA ${ }^{11}$ DE LA EDUCACIÓN FÍSICA}

Ahora bien, en los párrafos que preceden a estas líneas pueden verse a cada paso la afirmación de que en estos sucesos de la educación física se produjeron los elementos que se reproducirán, con distintas intensidades, en la Educación Física. Tomemos por caso la redacción del Documento oficial divulgado con motivo del Cincuentenario de la Ley de Educación de 1884: publicado en 1938 por el Consejo Nacional de Educación, las memorias reflejan los principales aciertos y deudas pedagógicas del medio siglo transcurrido. Para el caso específico de lo que por aquel entonces ya era la Educación Física, el Consejo entendía que en las "clases de ejercicios físicos" debía procurarse una acción que discipline física y psíquicamente, atenderse la influencia higiénica y adecuarse los ejercicios a criterios fisiológicos (Argentina, 1938: 134-135). Es decir, principios científicos puestos en práctica en forma de justificaciones higiénicas y fisiológicas, profundización de la sumisión de lo físico a lo psíquico y lo intelectual: la Educación Física "buscará así completar la disciplina física que es escasa en general en todos los niños para llegar a la disciplina psíquica" (1938: 136). Esto muestra a las claras que una vez cumplida la necesidad de formar la identidad nacional de fines de siglo XIX y principios del XX, el control del cuerpo en las escuelas, además de posibilitar el desarrollo intelectual, permitirá la disciplina psíquica, el gobierno de sí mismo incorporado como un gobierno de los otros. Para que la Educación Física se hiciera cargo de tamaña empresa fue necesario, además de las gimnasias, incorporar a los juegos como principal agente educador moral: entendidos como expresión del espíritu de los niños, su instrucción implicaba aprendizajes morales modernos y humanistas.

En la correlación que la Educación Física guarda con la Educación Moral, tenga el maestro presente que es en los juegos en donde se presenta desnuda el alma del niño con la revelación de todos sus instintos e inclinaciones, que es en ellos donde el maestro puede percibir más claramente todas esas diferencias de orden moral que tanto distinguen un niño de otro y que por lo tanto es en ellos donde se presenta la mejor oportunidad de corregir y encaminar no sólo el desarrollo físico del niño cuanto sus condiciones morales (Argentina, 1938: 137-138).

Respecto a los procesos que dieron origen a la Educación Física propiamente dicha en la Argentina, si se tuviese que datar con precisión cuál es la fecha en que nace, podríamos decir que es el 9 de Marzo de 1903, con la carta que Enrique Romero Brest, por ese entonces Inspector del área, le escribe a Juan Ramón Fernández, Ministro de "Justicia e Instrucción Pública":

Sólo me resta pedir al señor Ministro me permita proponer que en el programa de las Escuelas Normales figure esta materia con el nombre de 'Educación física', nombre más apropiado á la clase de estudios que comporta en estas Escuelas. Se trata en ellas de algo más que los simples Ejercicios físicos, desde el momento en que no solo se actúa sobre el individuo

11 La noción de conquista del objeto de estudio, el primero de los tres momentos por los que, según Bourdieu -quien sigue en esto a Bachelard-, atraviesa lo social indagado, supone una instancia de ruptura tanto con las maneras de concebirlo ligadas al sentido común cuanto con las tradiciones epistemo-metodológicas de las que emerge. (Bourdieu, 1992; Galak \& Gambarotta, 2011). 
como organismo físico sinó también como ser psíquico y moral y pues que se emprenden estudios extendidos de fisiología e higiene, lo que comprende algo más que la simple expresión 'Ejercicios físicos' (Romero Brest, 1903: 87-88).

Este hecho, que implica uno de los momentos disruptivos clave de la disciplina, no puede ser mentado si no es en relación con el contexto en el que se produce: la susodicha carta se realiza en el marco de la redacción del Programa sintético de Ejercicios Físicos para los "Cursos Normales de Educación Física", uno de los pioneros esfuerzos estatales para la instrucción de profesionales. Resulta significativo, en ese sentido, que la primera vez que se explicita la intención de nombrar el conjunto de prácticas educativas como "Educación Física", sea para construir su sistematización, su homogeneidad y su generalidad que la constituyan como práctica. A su vez, este hito -que marca el pasaje de la educación física a la Educación Física- muestra otra vez el dualismo en que está inmerso, que escinde al cuerpo de la psiquis, lo intelectual o lo moral. Pero también su rol utilitario, por el cual pretende enseñar contenidos que ni son autotélicos ni aún responden a los potenciales intereses de la propia disciplina, sino que permiten aprehender la moral necesaria para reproducir mecanismos de (auto)control.

\section{LA NATURALIZACIÓN DE LO NATURAL}

En los distintos elementos que conforman la constelación de sentido predominante en este momento genético (aunque no sólo en él) de la Educación Física argentina puede detectarse, entonces, el impacto de las categorías de pensamiento y acción propias de la sociedad moderna, retomando así ese hilo que ha atravesado nuestras consideraciones sobre la disciplina, remitiéndonos a la educación en general y, de ella, a la sociedad moderna en su conjunto. Estas categorías pueden englobarse (no sin un cierto simplismo, mas no por ello desacertadamente) como enraizadas en la lógica cartesiana, que primero escinde y luego subordina la res extensa a la res cogitans, viéndolas como dos entidades separadas y dividiéndolas en inferior y superior. Semejante concepción, propia de la sociedad moderna, es abordada aquí especialmente en el modo en que ella es tematizada por Elias $(1989,1992)$-quien en parte sigue en esto una matriz de pensamiento weberiana o, si se quiere, aborda la contracara del proceso de racionalización estudiado por Weber (Weber, 2003)-, en su inscribirla en el marco del proceso civilizatorio moderno. Elias se enfoca en dar cuenta de los avances de una lógica del (auto)control que subyace a semejante proceso, así como su concomitancia con la creciente monopolización de la coacción física a manos de un actor central (el Estado). Y, en este proceso, una dimensión clave, que es también central para nuestro análisis, está dada por cómo ese control no sólo es producido a partir de instancias externas, sino que también se da un proceso de introyección de tales controles, por el cual una instancia del propio agente (la superior) ejerce el control sobre la otra (la inferior), con la alienación que esto entraña. ${ }^{12}$

12 Si en Marx (2000) la lógica del capitalismo lleva a que el trabajador aliene su capacidad de trabajar, percibiéndola como una cosa más en el mundo de las cosas que, como tal, puede intercambiarse en el mercado, aquí encontramos como se aliena no ya una capacidad sino todo aquello "otro" a la res cogitans, al espíritu o al alma. Para un desarrollo de esta cuestión puede consultarse Gambarotta, 2010. 
A partir de esto podemos percibir los principios de (di)visión que se ponen en juego en la Educación Física argentina, a través de la cual abordamos algunos rasgos centrales de la educación, concibiendo a esta última como via regia para interrogarnos acerca de un hilo clave de la trama cultural de la sociedad moderna. Pues, y he aquí el quid de la cuestión, no sólo la educación escolar es una institución de carácter fundamentalmente moderno, sino que también es central para la reproducción de su lógica cultural. A lo que se agrega el que ella apunta no sólo a las mentes de los agentes, sino también a sus cuerpos (estableciendo esta distinción en el mismo instante en que la plantea), esto es, inscribiendo dicha lógica en sus esquemas de dotación de sentido incorporados, instalándolo en el entrelazamiento de nomos y doxa. Este enfoque general, que ha sobrevolado al conjunto de este escrito, se encontrará ahora particularmente presente, en tanto es a través de él que podremos extraer nuestras conclusiones sobre la Educación Física argentina, así como concebir otros modos de pensar la educación de los cuerpos.

De allí que nuestro interrogante sea, centralmente, acerca de los principios de (di)visión que hunden sus raíces en el mentado entrelazamiento, los cuales se encuentran en acto en el proceso socio-histórico que conduce a la génesis de la Educación Física argentina, pero cuya historicidad se petrifica, se "naturaliza". O, más precisamente, esa Educación Física no sólo produce la naturalización de un particular "arbitrario cultural" acerca del cuerpo, de su educación, etcétera, sino que ella misma, la manera en que desde ella se dota de sentido a estas cuestiones, es ya un producto de ese entrelazamiento de nomos y doxa naturalizado. Frente a esto, aprehender los mecanismos que (re)producen esta lógica social puede constituirse en una de las vías (no la única, pero sí una de ellas) por la que llevar adelante el esfuerzo en pos de hacer "saltar por los aires" tales mecanismos.

\subsection{EL CONTROL DEL DES-CONTROL}

A partir de nuestro trabajo sobre los discursos que se encuentran en la génesis de la Educación Física argentina, hemos destacado dos nodos problemáticos, vinculados entre sí, que resultan claves en el proceso social e histórico de substancialización y eternización que aquí estudiamos. Nos referimos, por un lado, a la reducción de lo corporal a lo físico y, por el otro, a la concepción de la educación como integral; ejes sobre los cuales recae el análisis del presente apartado. En torno al primer nodo, cabe comenzar destacando que dicha reducción conlleva, a su vez, una concepción de la educación de los cuerpos centrada en los "ejercicios físicos" o en las "actividades físicas"; lo cual, al mismo tiempo, estrecha el debate educacional a la tarea de dirimir cuáles son los mejores, los más eficaces ejercicios en pos de educar el físico de los niños en edad escolar, simplificando así al cuerpo como "máquina biológica", al mismo tiempo que evoca su rol utilitarista. Romper con esta concepción naturalizada de lo corporal como físico constituye un primer gesto necesario para la reintroducción de la historia en estos principios de (di)visión des-historizados, abriendo así la lucha en lo político allí donde el entrelazamiento de nomos y doxa la clausura.

Ruptura que, a nuestro entender, retoma la tensión subyacente a la noción de "Educación Física", tal como se la planteaba en este momento genético de la disciplina y que hoy ha quedado aplanada bajo la amnesia (en el sentido que Bourdieu en La dominación masculina la concibe) de los procesos y batallas sociales que dieron lugar a dicha 
génesis. Se trata de retomar, entonces, la tensión entre términos percibidos y apreciados como opuestos, en pos de reabrir aquellas batallas que la amnesia de la historia clausura, buscando en definitiva generar las grietas que nos permitan reflexionar en torno a un nuevo concepto de la educación de los cuerpos. Es esa tensión la que late en las palabras de Romero Brest que hemos citado, en la ruptura que -en ese momento- implicaba el proponer el nombre "Educación Física" frente a la expresión "ejercicios físicos"; pues a dicho nombre, aun cuando esté presente un sentido fisicalista, subyace la concepción de que allí hay algo educable. En definitiva, que ese "físico" -común a ambas designaciones- no es un objeto más en el mundo de los objetos, lo cual implica un principio de agrietamiento del dualismo cartesiano en su versión más extrema, en tanto desde la manera de ver y dividir el mundo de este último allí hay un mero objeto, por lo que no habría nada que quepa ser "educado". Es decir que si bien el cambio de denominación impulsado por Romero Brest mantiene la alusión al "físico" -con todo lo que ello entraña según lo aquí planteado-, hay en su propuesta una búsqueda por romper con aquella concepción que pone el foco en el "ejercicio" de ese físico, en la repetición de determinadas "actividades" que no necesariamente implican una transmisión de "saber". Es a darle un lugar a ese saber, a su transmisión, o si prefiere a un cuestionamiento pedagógico de ese saber, lo que late en las palabras que le dirige al Ministro.

Ahora bien, ese dualismo cartesiano pervive aún en esas mismas palabras de Romero Brest; pues se reivindica el carácter "educativo" de esta práctica en tanto no sólo apunta al organismo físico sino también a lo psíquico y a lo moral. Vemos así como ese agrietamiento no deja de enmarcarse en la lógica del discurso de la educación integral, con una corporalidad concebida únicamente como físico, de cuya educación se hace, a su vez, un camino por el cual alcanzar un objetivo otro, "superior": la educación psíquicomoral de los niños. Y, según Romero Brest, es porque impacta en estas instancias que merece denominarse como "educación", es por apuntar a algo distinto de aquello que se concibe como "físico" que esta (nueva) disciplina "comprende algo más que la simple expresión "Ejercicios físicos"”.

Esto nos lleva a nuestro segundo nodo problemático, el marco general dado por la educación integral, el cual conforma el terreno en el que hunde sus raíces el proceso genético de la Educación Física argentina. Es a partir de la lógica que aquella pone en juego que se hace de esta última -tal como ya apreciamos en las palabras de Romero Brest- una vía por la cual alcanzar el objetivo de educar a las instancias percibidas y apreciadas como "superiores": las del espíritu, la intelectual y moral. Es a partir de esta concepción general que se conforman las "técnicas" que se pone en juego en la Educación Física argentina, el conjunto de modos de hacer -que no distinguen forma de contenido, sino que aprehenden el entrelazamiento entre ambos- que allí se implementa y del cual pueden remarcarse dos rasgos centrales: en primer lugar, cómo la mencionada percepción de la Educación Física hace de ella no un fin en sí mismo, sino una vía por la cual alcanzar un objetivo que se sitúa más allá de lo "físico"; es, en última instancia, un medio para alcanzar un fin más elevado. Sobre esta base puede captarse cómo el conjunto de técnicas que aquí predomina es de carácter instrumental, favorecido por el proceso de alienación de lo corporal que hace de éste una cosa a ser controlada, es decir por una percepción del cuerpo cosificada y, como tal, pasible de manipulaciones instrumentales. Todo lo cual sitúa como parámetros centrales para establecer ("medir", "cronometrar", "cuantificar") la calidad de los ejercicios a su efectividad y eficiencia. ¿En pos de qué? 
Pues del objetivo al que la educación integral apuesta y del que la Educación Física es un medio.

En segundo lugar, cómo la tarea que ella tiene es, casi explícitamente, la de brindar una descarga de energía frente a las tareas áulicas, tal como se desprende el análisis de los artículos $1^{\circ}$ y $14^{\circ}$ de la Ley $\mathrm{N}^{\circ} 1.420$. Se apunta, entonces, a erradicar del aula -espacio principal de la educación- aquello que des-controlaría esa clase. Así, se controla semejante amenaza a través de la generación de un ámbito específico que resulte propicio para esa descarga, siendo ése, precisamente, el rol de la asignatura de Educación Física o bien del recreo -y también del canto, abriendo una dimensión estética como posible línea por la cual seguir avanzando en estas cuestiones-. Es allí donde puede descargarse todo aquello que atañe a lo "natural" en el ser humano, a lo apreciado como bajo y que, como tal, obstaculiza el desarrollo de las instancias consideradas superiores, a las que se aboca el trabajo en el aula.

Pero aclaremos inmediatamente que lo "natural" aquí no ha de entenderse como dado a priori y establecido de una vez y para siempre, sino que se define a partir del propio discurso de la educación integral -refrendado por el de la Educación Física-, por ser aquello que ella excluye, que señala como algo a ser controlado (y sólo en relación con semejante concepción es que puede pensarse que lo "natural" sea una suerte de depositario del "des-control"). Ésta es la manera en que se dota de sentido a los impulsos de los niños, pero también a sus movimientos o, al menos, a aquellos que atentan contra las tareas áulicas, con su vista al frente y cada niño acomodado en su pupitre. Vemos así como, en el marco más amplio de la educación integral, la Educación Física se configura como parte del esfuerzo por controlar la des-controlada "naturaleza", reduciendo lo corporal a lo físico y abordándolo técnico-instrumentalmente.

Esto se encuentra aún más explícitamente planteado en la siempre explícita pluma de Sarmiento, quien sostiene la inclusión de la gimnástica en las escuelas sobre la base de que ella entraña un "factor de disciplina" a través del cual se pueden controlar los "impulsos primitivos" del niño en pos de su educación moral. ${ }^{13}$ Nuevamente nos hallamos ante una mirada integralista de la educación, que sitúa como tarea fundamental de la Educación Física (aunque sería mejor decir, de aquello que terminó constituyéndose como Educación Física, después de un proceso genético que implicó luchas con otras maneras de entender la educación de los "físicos") el control de eso otro que es percibido como fuente del des-control. Tarea que ha de realizarse no sólo por medio de lo que con Elias (1989) podemos concebir como una "instancia externa" (la escuela), sino que esa misma institución es también protagonista (o co-protagonista, junto con la familia) de la introyección de ese control por parte de los propios agentes, que se instauran así como "controladores" de su propio "des-control" (con la alienación que ello entraña). Introyección a la que subyace, en última instancia, la inscripción en los cuerpos de los principios de (di)visión en los que se enraíza esta lógica social, esto es, la incorporación

13 Lo cual puede hallar sustento argumentativo en la prédica de Spencer quien sostenía que más importante que la gimnasia (contenido por ese entonces casi exclusivo de la educación física) era la incorporación de la enseñanza de los juegos, entendiendo que estos últimos eran superiores a la primera por el factor moral que ponían "en juego", de allí que su enseñanza sirviese (según esta concepción) para dominar la moral de los niños (Spencer, 1946: 248-250). 
de ese modo de dotación de sentido que lidia con un "físico" a ser instrumentalmente abordado, controlado, gobernado.

Es en este punto donde vuelve a emerger el vínculo con el proceso civilizatorio ${ }^{14}$ tal como Elias (1989, 1996, Elias y Dunning, 1992) lo conceptualiza, no sólo por la doble vía a través de la cual se ejercen estos controles, sino también por cómo es el mismo proceso (civilizatorio) el que constituye ese "otro", esa "naturaleza" que es el objeto del control (externo e interno). En efecto, Elias muestra cómo muchos rasgos que hoy consideramos como propios de eso "natural" que hay en nosotros -nuestros sentimientos de asco o de pudor, pero también la manera en que lidiamos con nuestras pasiones o con nuestras necesidades básicas- son un producto del proceso civilizatorio y, como tales, socio-históricamente generados. En este marco general, característico de la sociedad moderna, la Educación Física encuentra su lugar en tanto engranaje del mecanismo de control de aquello que la educación intelectual y moral define como fuente del "descontrol". Definición socio-histórica y cultural cuya fuerza proviene, principalmente, de presentarse investida con los ropajes de lo "natural", de que se perciba a través de esas lentes a aquellas prácticas que no pueden tener lugar dentro del aula, que sólo pueden situarse fuera de ella pero en función de lo que en ella acontece, brindando un espacio de descarga que resulta benéfico para las actividades áulicas.

\subsection{EL DES-CONTROL DEL CONTROL}

Sin embargo, este proceso no se detiene aquí; retomando el planteamiento bourdieuano, que ha atravesado a este escrito, podemos señalar cómo es justamente la petrificación de estos principios de (di)visión, el entrelazamiento de nomos y doxa que allí tiene lugar, lo que genera la doble naturalización de lo socio-histórico que acontece en la Educación Física argentina (pero que remite más allá de ella, a la educación en general y a la sociedad en su conjunto). Y esto tiene lugar tanto en las cosas, en instituciones como la escuela, en el modo en que ella plantea este juego, cuyas reglas condicionan la manera en que los agentes lo juegan, como en los cuerpos, a través de la incorporación de estos principios de (di)visión en los agentes, cuyo modo de jugar este juego condiciona la conformación de las reglas del mismo.

Es esta doble naturalización, entonces, la que subyace al modo de (re)producción de los cuerpos, que se encuentra presente, de manera dominante, en la Educación Física argentina, y que esta última pone en acto en sus diversas instancias, pero sin que esto implique necesariamente una consciencia por parte de los agentes sociales involucrados (profesores, diseñadores de currículos, etcétera) de las consecuencias de sus prácticas, antes bien si estos principios de (di)visión se encuentran incorporados es porque ellos también subyacen a la manera en que tales agentes perciben, aprecian y actúan en este ámbito. En definitiva, ese entrelazamiento de nomos y doxa delimita el espacio de lo

14 No casualmente esto tiene lugar en el marco dado por el dominio de la generación del 80 en la Argentina, que es también uno de los momentos claves del fin de las luchas armadas internas en dicho país, con el consecuente proceso de monopolización de la fuerza física por parte del Estado Nacional. De más está decir que la historia del fin de tales luchas es mucho más basta y compleja, pero sin dudas éste fue uno de sus mojones claves, el cual puede simbolizarse en torno a la federalización de la Ciudad de Buenos Aires y de los ingresos de su aduana. 
pensable y lo posible dentro de los márgenes de la Educación Física argentina, relegando a impensable o imposible todo lo que se sitúe por fuera de su lógica. ${ }^{15}$

Es dando cuenta de este modo de (re)producción de los cuerpos que puede aspirarse a transformarlo, y con tal fin hemos señalado algunos de los elementos centrales que conforman su constelación, basada en una concepción dual (de raíz cartesiana) que se complementa con la reducción a lo "físico", técnico-instrumentalmente abordado. ${ }^{16}$ Sobre esta base se produce la erradicación de (lo que desde estos mismos principios de di-visión se considera) lo "natural", y es justamente esto lo que se "naturaliza". Así, el control de lo natural conduce a la (doble) naturalización del control; más aún, el control del descontrol tiene por corolario y punto cúlmine al des-control del control. ${ }^{17}$ Precisamente, a que sea este último movimiento el que subyace al modo que rige la (re)producción de los cuerpos en la Educación Física argentina, modo que se presenta a los agentes como una lógica extraña a pesar de que ellos la (re)instauran con sus prácticas. Más aún, como la única lógica posible para pensar la educación de los cuerpos, pues las propias categorías a partir de las que se dota de sentido a estos procesos son un producto (a la vez que productoras) de esos mismos procesos. Estamos, de esta manera, en el núcleo de la dominación simbólica, dando cuenta del proceso por el cual se reconoce a esta particular concepción de la educación de los cuerpos, que lleva el nombre de Educación Física, como la legítima; reconocimiento que, en parte, se asienta en el desconocimiento de los procesos socio-históricos que llevaron al triunfo de estos principios de (di)visión y no de otros. Es sobre esta base que puede producirse la transmutación del nomos sociohistórico y culturalmente arbitrario en necesidad de la naturaleza.

\section{DE LA EDUCACIÓN FÍSICA A LA EDUCACIÓN CORPORAL}

Si estos planteamientos no presentan un panorama ajeno al de la Educación Física actual es justamente por la acción de la reproducción. Esta frase inicial, que funciona al mismo tiempo tanto como conclusión general cuanto como diagnóstico transversal de la situación dominante en el presente de la Educación Física argentina, cobra dimensión particular al permitirnos enfocar la mirada en los mecanismos por los cuales dicha reproducción se sostiene. En este sentido, la petrificación de lo socio-histórico que se produce en la instauración de la dominación simbólica que la educación de los cuerpos supone, implica la clausura de lo político y sus luchas. Es frente a esto que surge el desafío de esforzarnos por abrir la lucha en lo político en torno a estas cuestiones, es decir, por disrumpir críticamente el entrelazamiento de nomos y doxa junto con la doble naturalización a que da lugar. En esta dirección puede percibirse la necesidad de orientar nuestras prácticas de investigación hacia el planteo de una educación de los cuerpos que interrumpa la continuidad de la lógica que atraviesa a la ley $\mathrm{n}^{\circ} 1.420$ así como a la

15 Puede encontrarse un trabajo más detallado sobre estas categorías en Bourdieu $(1995,1999)$ y Gambarotta (2012).

16 Para profundizar sobre la relación entre físico y cuerpo y la permanencia de esa concepción en la prácticas de la Educación Física puede ampliarse con Galak (2009).

17 Para un desarrollo de esta tensión, que cabe concebir como una "dialéctica aporética", entre control y descontrol en referencia a otro particular modo de producción de corporalidad puede consultarse Gambarotta (2011). 
concepción de la educación integral, introduciendo allí una discontinuidad que haga "saltar por los aires" los mecanismos que reproducen a este particular modo de los cuerpos.

Entendemos que la clave está en la doble dimensión de, por un lado, la reducción que tiene lugar en este modo de construir los cuerpos como un elemento meramente físico, y, por el otro, el rol que se le asigna a la Educación Física como medio para conseguir ciertos fines particulares y ulteriores. En este marco, la desarticulación de estos mecanismos pasa por romper con estas concepciones, dando lugar a un cuestionamiento a la Educación Física tal como se la ha entendido, a partir de reconfigurar la constelación de elementos que se ponen en juego en las prácticas de la disciplina. Esto implicaría reflexionar acerca de las construcciones que surgen en relación con la ley 1.420 y los planteos en la que ésta se sustentaba -tales como los de Spencer y Sarmiento-, poniendo especial énfasis en el agrietamiento de la lógica que hace de la educación física un elemento (subordinado dentro) de la educación integral.

Esto implica no sólo cuestionar el dualismo propiamente moderno sino también erradicarlo de las prácticas disciplinares, lo cual conlleva un abandono de la concepción fisicalista para centrar la educación en el cuerpo. El pasaje que planteó Romero Brest de la denominación de "ejercicios" a "educación" representa un primer gesto en esa dirección, sin embargo ambas instancias replican la alusión a lo físico, de allí que se mantenga en su trasfondo una lectura integralista accesoria a lo moral e intelectual. Por ello, profundizar ese gesto requiere pensar una educación no ya del físico, sino de los cuerpos.

Es decir, pensar una educación corporal como vía de disrumpir con la continuidad que la tradición naturalizada de la distinción propuesta por la educación integral implica, dando lugar a la emergencia de otras técnicas no-instrumentales y no-utilitarias. Por caso, si pudo concebirse la idea de un "proletariado intelectual" o un "proletariado manual" es porque en el marco de esa unión que compuso la educación integral se reprodujeron técnicas instrumentales. En cambio, la propuesta de pensar una educación, más que del físico, del cuerpo, nos lleva a pensar la posibilidad de técnicas no-instrumentales y noutilitarias a ser puestas en práctica en la adherencia de saberes (producto de la relación entre disciplinas). Por esto mismo, la educación corporal apunta a provocar una subversión simbólica del orden establecido por la educación integral, en el que la división en partes que componen una cosa ya no sea posible, que la sumatoria de partes no hagan la cosa, sino más bien que producto de la transversalidad (de contenidos, de saberes, de prácticas) resulte el acto educativo.

La dominación simbólica, que tiene como una de sus instancias una determinada manera (legítima) de educar el cuerpo, se sustenta en la violencia simbólica propia de toda educación. De ninguna manera debería leerse entonces estos párrafos como una crítica radical a toda forma de educación sino, más bien, como una crítica a toda educación radical que, fundada en su fundamentalismo, no permite la posibilidad de diluir los dualismos, dando lugar a una relación otra entre los elementos de esa constelación. Porque, como hemos visto, aun la ilusión de poder elegir entre uno de los dos polos (opuestos) de toda binariedad a la que se resume lo cotidiano, producto de la (di)visión del mundo social, constituye, en definitiva, un modo de permanecer encerrados en una (sola) lógica. Frente a ello, consideramos que en la potencialidad de la transmisión, que toda educación supone, se hallan las posibilidades de sortear los límites que la Educación Física entraña; en este sentido no planteamos una crítica a la educación sino más bien 
una apuesta por la misma, pero corriendo el eje del nomos centrado en el físico a otros que den cuenta del cuerpo.

\section{REFERENCIAS BIBLIOGRÁFICAS}

Aisenstein, Á. y Scharagrodsky P. (2006). Tras las huellas de la Educación Física Escolar Argentina. Cuerpo, género y pedagogía. Buenos Aires: Prometeo.

Argentina (1884). Ley n ${ }^{\circ} 1.420$ de Educación Común, aprobada el 8 de Julio de 1884.

Argentina (1938). Cincuentenario de la Ley 1420. Tomo II: memorias sobre el desarrollo de las escuelas primarias desde 1884 a 1934. Consejo Nacional de Educación, Buenos Aires. Argentina.

Bourdieu, P. (1992). Thinking about limits. Theory, Culture \& Society, n. 9, 37-49.

Bourdieu, P. (1995). Las reglas del arte. Génesis y estructura del campo literario. Barcelona: Anagrama.

Bourdieu, P. (1999). Meditaciones pascaliana. Barcelona: Anagrama.

Bourdieu, P. (2001). El campo político. La Paz: Plural editores.

Bourdieu, P. (2007). La dominación masculina. Barcelona: Anagrama.

Bourdieu, P. (2008). ¿Qué significa hablar? Economía de los intercambios lingüísticos. Madrid: Akal.

Crisorio, R. (2009). Educación Física. En R. Crisorio, y M. Giles (Dirs.), Educación Física. Estudios críticos en Educación Física (pp.45-58). La Plata: Al Margen

Cristiani, R. (1967). Reseña histórica del cuerpo de Gimnasia y Esgrima del Ejército y su proyección en la vida nacional. Algunos aspectos de su evolución entre 1897-1960. Comando en Jefe del Ejército, Dirección de Estudios Históricos. Buenos Aires: Argentina.

Dallo, A. (2007). Enrique Romero Brest. Boletín de la Academia Nacional de Educación, Buenos Aires, $\mathrm{n}^{\circ} 69$.

Elias, N. (1989). El proceso de civilización. México: Fondo de Cultura Económica.

Elias, N. (1996). La sociedad cortesana. México: Fondo de Cultura Económica.

Elias, N. y Dunning, E. (1992). Deporte y ocio en el proceso de la civilización. México: FCE.

Galak, E. (2009). El cuerpo de las prácticas corporales. En R. Crisorio, y M. Giles (Dirs.), Educación Física. Estudios críticos en Educación Física (pp.271-284). La Plata: Al Margen

Galak, E. (2010). El concepto cuerpo en Pierre Bourdieu. Un análisis de sus usos, sus límites y sus potencialidades, Tesis de Maestría en Educación Corporal. Universidad Nacional de La Plata: Argentina.

Gambarotta, E. (2011). La dialéctica aporética del modo de corporalidad pugilístico: el control de lo natural y su descontrol. En V. D'hers, y E. Galak (comp.), Estudios sociales sobre el cuerpo: prácticas, saberes, discursos en perspectiva (pp.20-37), ESE.

Gambarotta, E. (2012) Hacia una teoría crítica reflexiva. Una lectura sobre los aportes de Max Horkheimer y Pierre Bourdieu. Cuestiones de Sociología, n.7/8, 276-293.

Galak, E. y Gambarotta, E. (2011). Conquista, confirmación y construcción del cuerpo. Una propuesta para el estudio de las prácticas corporales a partir de la epistemología de Pierre Bourdieu. Revista Brasileira de Ciências do Esporte, vol. 33, n.4.

Gambarotta, E. (2010). La dialéctica aporética entre cuerpo y sí mismo: Una lectura de Dialéctica de la ilustración en clave política. Intersticios. Revista Sociológica de Pensamiento Crítico, vol. 4, n.1, 147-161.

Instituto Nacional Superior De Educación Física (1923). (INSEF) Colación de grados de 1923. Publicación costeadas por los profesores egresados. Buenos Aires: Argentina.

Lukács, G. (1971). Teoría de la novela. Barcelona: Edhasa.

Marx, K. (2000). El Capital. México: Fondo de Cultura Económica.

Merleau-Ponty, M. (1957). Las aventuras de la dialéctica. Buenos Aires: Leviatán. 
Narodowski, M. y Manolakis, L. (2001). Estado, mercado y textos escolares. Notas históricas para un modelo teórico. Revista Educación y Pedagogía, vol. XIII, n.29-30, 27-38.

Pourteau, S. (1897). Breves reflexiones sobre la Educación Física. Buenos Aires: Imprenta Helvetia.

Rodríguez Giménez, R. (2010). Notas para una investigación sobre pedagogía y biopolítica. Educación Física y Deporte, vol. 29, n.2, 215-223.

Romero Brest, E. (1903). Cursos Normales de Educación Física (sus resultados). Buenos Aires: Librería y Casa Editora de Nicolás Marana.

Romero Brest, E. (1913). La educación física argentina. Congreso internacional de París de 1913. Buenos Aires: Librería e Imprenta Europea de Rosas.

Saraví Riviere, J. (1985). Historia de la Educación Física Argentina. Siglo XIX (notas para su elaboración). Buenos Aires: Impresiones Agencia Periodística Cid.

Sarmiento, D. (1900). Obras de D. F. Sarmiento. Buenos Aires: Imprenta Mariano Moreno.

Scharagrodsky, P. (2004). El padre de la Educación Física Argentina: fabricando una política corporal generizada (1900-1940). Revista Perspectiva, Revista do Centro de Ciencias da Educaçao, vol. 22, 83-119.

Scharagrodsky, P. (2010). Nuestra educación física tiene raíz militar: había que controlar los cuerpos, entrevista por Claudio Martyniuk. Diario Clarín, 28/05/2010.

Spencer, H. (1946). Educación intelectual, moral y física. Buenos Aires: Albatros.

Weber, M. (2003). La ética protestante y el espíritu del capitalismo. En Obras selectas, Buenos Aires: Distal. 
